\title{
Drilling of CFRP with Reduced and Non-Directional Heat-Affected Zone by Using 3-kW CW Single-Mode Fiber Laser
}

\author{
Yuya Takubo*, Daisuke Sekine, Junji Sato, Keisuke Uchiyama, Shinya Ikoma and Yoshio Umeda \\ Optical Technologies R\&D Center, Fujikura Ltd., Japan \\ *Corresponding author's e-mail: yuya.takubo@jp.fujikura.com
}

\begin{abstract}
High-precision drilling of carbon fiber reinforced plastic (CFRP) using a 3-kW continuous-wave (CW) single-mode fiber laser has been demonstrated. Holes with a diameter of $6.4 \mathrm{~mm}$ have been successfully drilled on a 2-mm-thick thermoset CFRP. The laser was scanned at a scanning speed of $10 \mathrm{~m} / \mathrm{s}$ with a galvanometer scanner by a multi-pass method with a time interval of $20 \mathrm{~ms}$. The processing time was 2.7 seconds, which was equivalent to that in mechanical processing. By optimizing the conditions of scanning and gas injection, the width of heat-affected zone (HAZ) was controlled to about $100 \mu \mathrm{m}$. Although a HAZ tends to expand in the direction of carbon the fibers, the zone was well-controlled in all directions. The results show the potential of the high-power CW singlemode fiber laser for the application to high speed and high quality drilling of CFRP.
\end{abstract}

DOI: $10.2961 / \mathrm{jlmn} .2020 .03 .2006$

Keywords: CFRP, drilling, single-mode, fiber laser, galvanometer scanner, heat-affected zone

\section{Introduction}

The demand for CFRP as a lightweight and highstrength material is growing especially in the aviation and automotive industries. Although the processing with machine tools is the most general method for trimming and drilling CFRPs, tools used for those processing are easily damaged due to the high strength of CFRPs. As worn tools lead to the degradation of quality, they need to be frequently replaced. In recent years, laser processing has attracted attention as a wear-free method for processing CFRP. The biggest problem in laser processing of CFRP is the generation of HAZs. Using a pulsed laser or ultrashort pulsed laser is an effective way of minimizing the HAZs [1-5]. However, the low average power of those lasers lead to relatively long processing time. Processing with a singlemode laser and a galvanometer scanner is a promising solution to achieve a short processing time and small HAZ simultaneously [6-10]. We reported high speed cutting of CFRP using a 3-kW single-mode fiber laser and galvanometer scanner [11]. A 3-mm-thick CFRP plate was successfully cut at an effective cutting speed of $7.8 \mathrm{~m} / \mathrm{min}$ and a small HAZ of less than $100 \mu \mathrm{m}$ was obtained.

Joining CFRPs together or CFRP and another material is also an important matter in industrial application. Mechanical fastening and adhesive bonding are general joining methods in the industries. For mechanical fastening with rivets and bolts, taking mass production in account, many holes need to be drilled in a short time. Therefore, laser drilling of CFRP is worth employing as well as laser cutting. In the laser drilling of CFRP, the problem is that the direction of expansion of HAZ is different from that in cutting. Since a HAZ tends to expand in the direction of the carbon fibers due to its high heat conductivity, the shape of it becomes an ellipse [12]. Reducing the width of the HAZ in all directions requires the optimization of the conditions of scanning and gas injection.
In this paper, we demonstrate the drilling of 2-mm-thick thermoset CFRP using a 3-kW single-mode fiber laser and a galvanometer scanner. As a single-mode fiber laser can achieve a high power density and small spot diameter, energy needed for processing is effectively provided. In addition, high speed scanning by a galvanometer scanner will reduce the width of HAZ. Considering a balance of a processing time and the width of HAZ, an output power of 3 $\mathrm{kW}$ was adopted. The laser was scanned at a scanning speed of $10 \mathrm{~m} / \mathrm{s}$ and a time interval of $20 \mathrm{~ms}$. Holes with a diameter of $6.4 \mathrm{~mm}$ were successfully drilled in $2.7 \mathrm{sec}$ onds, which was the same duration as in mechanical drilling. By the optimization of the conditions, the width of HAZ was controlled to about $100 \mu \mathrm{m}$ in all directions. While the processing time was shorter than that in processing using pulsed lasers $[5,12]$, the HAZ was limited to relatively small width. The results show the capability of the high power single-mode fiber laser for high-speed highquality drilling of the CFRP.

\section{Processing conditions}

Figure 1 (a) shows the processing test setup. The inhouse manufactured $3-\mathrm{kW}$ single-mode fiber laser was connected to the galvanometer scanner via the laser delivery cable. The optical magnification of the galvanometer scanner was 3 and the corresponding spot diameter was about $100 \mu \mathrm{m}$. The Rayleigh length of our laser setup was $5.6 \mathrm{~mm}$. The 2-mm-thick thermoset CFRP was selected as the workpiece. The CFRP plate consists of 7 layers made of unidirectional fabric layers, with fiber orientations of 0 degree and 90 degree. The gas injection nozzles were set above and below the CFRP plate. The nozzle on the upper side was set for cooling and removing the carbon fiber deposition, and the nozzle on the lower side was just for cooling. Figure 1 (b) shows the magnified image of the gas injection setup. The center of the nozzle was hollow and therefore the laser beam could pass through the nozzle. 
There were the outlets on the edges of the nozzles, and nitrogen gas blew out from the outlets toward the processing area. Such cylindrical nozzles have not been used for laser drilling of CFRP. When we used conventional nozzles, it was impossible to control the width of HAZs due to the gas flow with low velocity and non-uniformity. When using selected cylindrical nozzles, HAZs will be reduced in all directions. The definition of HAZ is different in researches $[13,14]$. In this report, HAZ stands for the area where the matrix material is vaporized and the carbon fibers are left, which is the same definition as matrix evaporation zone (MEZ) in [14].

The multi-pass method, in which a laser is scanned on the same position at high speed, is known to be an effective way of reducing the width of HAZ. We have adopted this method in the previous cutting experiment as well [11]. Prior to the processing test, we evaluated the performance of our galvanometer scanner at high scanning speed with low output power. We set the scanning speed to $10 \mathrm{~m} / \mathrm{s}$, which was the maximum speed of our galvanometer scanner to draw a 6.4-mm-diameter circle precisely. It takes about $2 \mathrm{~ms}$ to draw a circle in this condition. In the multipass method, the time interval is generally set between each scan. We optimized the interval for the first step to determining the scanning conditions. Taking the acceleration and deceleration of the scanner into account, we added the 1-lap scan without laser output before and after the scanning with laser output. The times for these scans were included in the time interval.

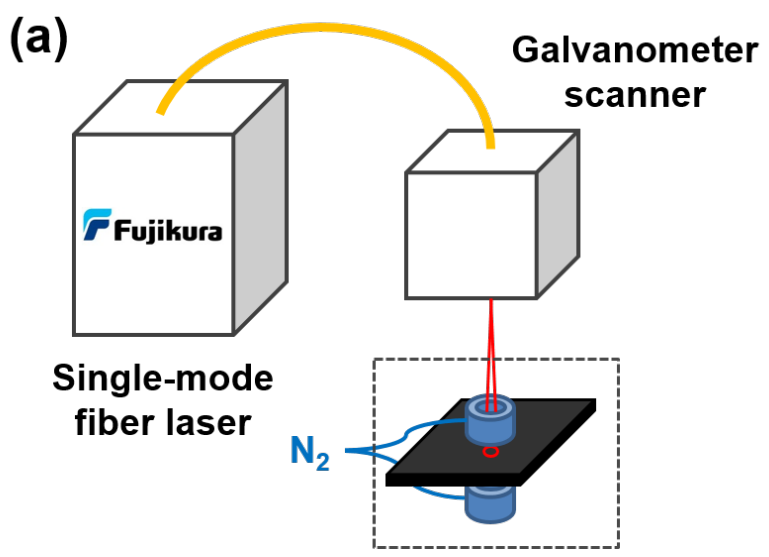

(b)

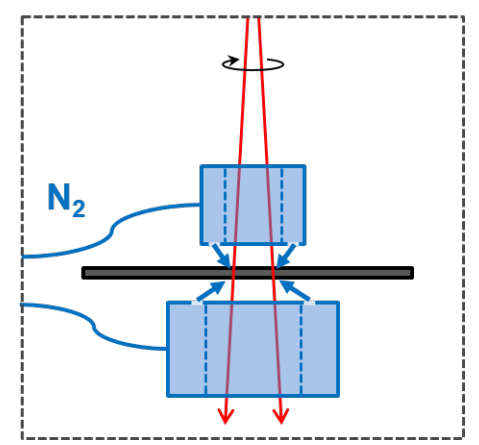

Fig. 1 (a) Processing test setup. (b) Gas injection setup.
Figure 2 shows the maximum width of HAZ versus the number of scans at the time interval of 0 to $20 \mathrm{~ms}$. The width of HAZ increased to more than $700 \mu \mathrm{m}$ after 30-time scans without interval, whereas it was limited to about 100 $\mu \mathrm{m}$ with a time interval of $20 \mathrm{~ms}$. As the interval is generally set to several hundred milliseconds to several seconds, our processing setup can control the HAZ with quite a short time interval.

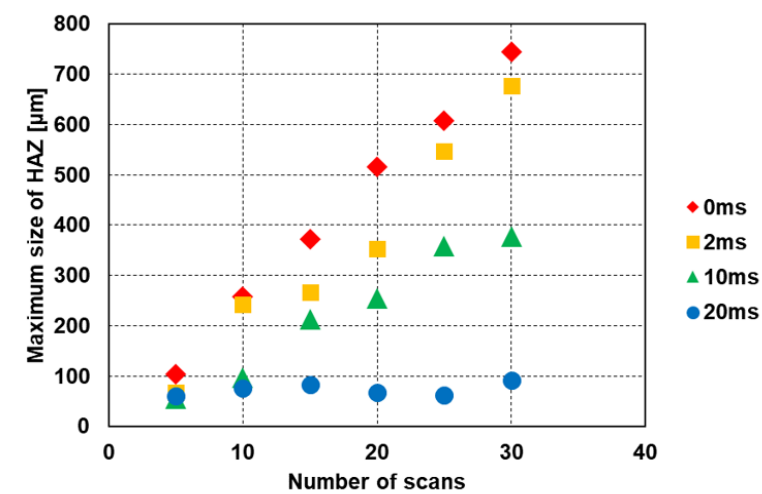

Fig. 2 Maximum width of HAZ versus the number of scans at each time interval.

To drill a hole in a short time, it is effective to process with a sufficient kerf width in removing the carbon fiber deposition [15]. Figure 3 shows the scanning position of the laser beam. The double concentric circles were drawn at each focal plane. The focal plane was shifted by $1 \mathrm{~mm}$ after a certain number of scans to improve processing efficiency. At the same time the focal plane shifted, the gap between the inner and outer circles was narrowed by $50 \mu \mathrm{m}$, which was the same value as in our previous cutting experiment [11].
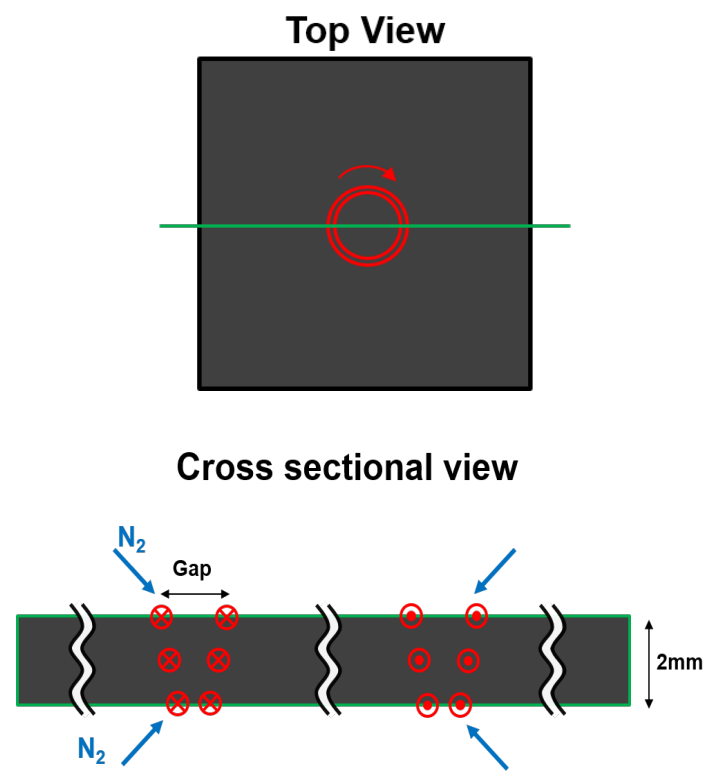

Fig. 3 Scanning position.

We set the gap between the circles at the surface to 150 $\mu \mathrm{m}$ and $350 \mu \mathrm{m}$, and compared the processing time. Figure 4 shows the kerf depth versus the number of scans in each condition. When the processing was started with the gap of $150 \mu \mathrm{m}, 108$-time-scans were needed, whereas it took only 
90 scans for $350-\mu \mathrm{m}$ gap. Although there was just a slight difference in the kerf depths, the processing time was shorter for $350-\mu \mathrm{m}$ gap. Figure 5 (a) and (b) show the cross-sectional images after 70 scans in each condition. The images indicate that the larger kerf width with the gap of $350 \mu \mathrm{m}$ leads to the short processing time. As seen in Fig. 5, one side of the kerf walls has deep HAZs. They will not be a problem since the HAZs are on the wall of the stub to be discarded.

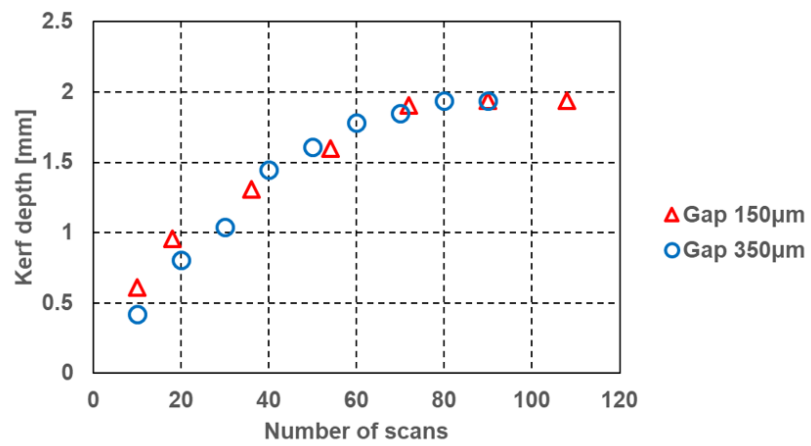

Fig. 4 Kerf depth versus the number of scans in each condition. (a)

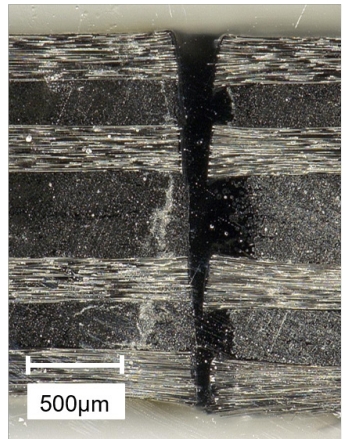

(b)

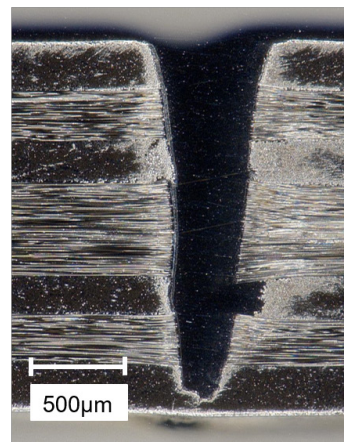

Fig. 5 Cross-sectional images after 70 scans with the gap at the surface of (a) $150 \mu \mathrm{m}$ and (b) $350 \mu \mathrm{m}$.

Table 1 shows the final scanning conditions based on the described experiment. We set the gap between the circles at the surface to $350 \mu \mathrm{m}$, and reduced it by $50 \mu \mathrm{m}$ as we shifted the focal plane by $1 \mathrm{~mm}$. The number of scans was set to 20,30 and 40 at each focal plane. As the kerf depth had reached to the bottom in the early phase of processing, we set more scans at the bottom. We carried out the drilling experiment with these conditions.

Table 1 Scanning conditions for drilling

\begin{tabular}{ccc}
\hline Focal position & $\begin{array}{c}\text { Gap of scanning } \\
\text { position }\end{array}$ & Number of scans \\
\hline $0 \mathrm{~mm}$ (surface) & $350 \mu \mathrm{m}$ & 20 \\
$1 \mathrm{~mm}$ & $300 \mu \mathrm{m}$ & 30 \\
$2 \mathrm{~mm}$ & $250 \mu \mathrm{m}$ & 40
\end{tabular}

\section{Processing test results}

Figure 6 shows the surface images of the processed CFRP plate. The 6.4-mm-diameter hole with good roundness was achieved. The CFRP plate of $2 \mathrm{~mm}$ thickness was successfully cut in 2.7 seconds, which was equivalent to that in mechanical processing. Although the processing time estimated from the scanning conditions was about 2 seconds, the performance deterioration of the galvanometer scanner at high scanning speed led to a bit longer processing time than estimated. Figure 7 shows the image obtained by a scanning electron microscope (SEM). The width of the HAZ was reduced to about $100 \mu \mathrm{m}$.

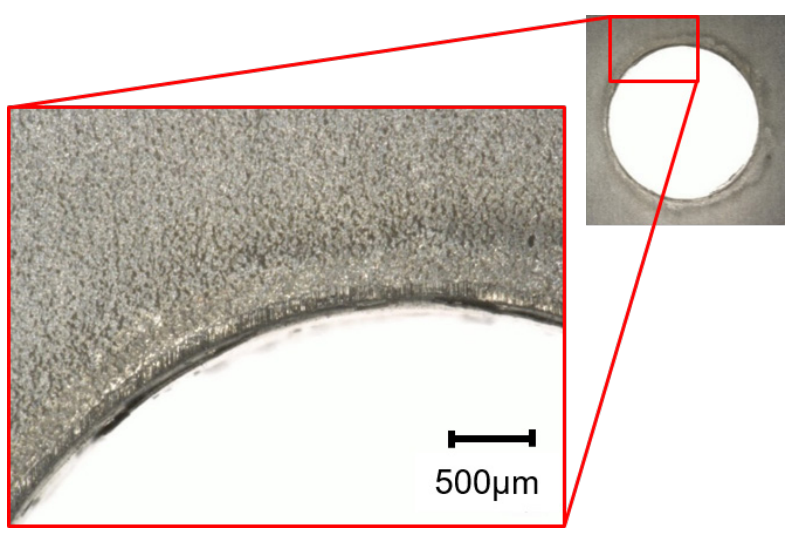

Fig. 6 Surface image of the processed CFRP plate.

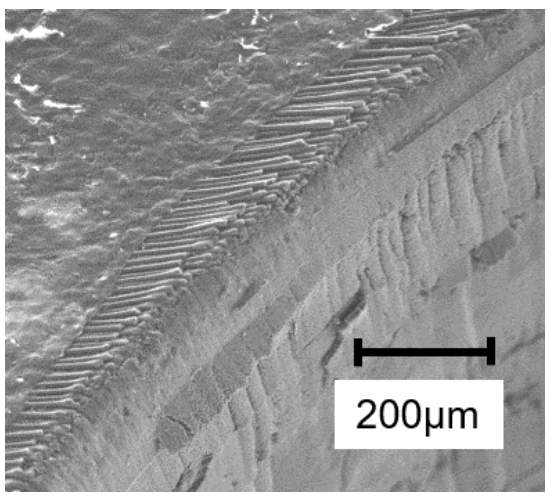

Fig. 7 SEM image of the CFRP near the surface.

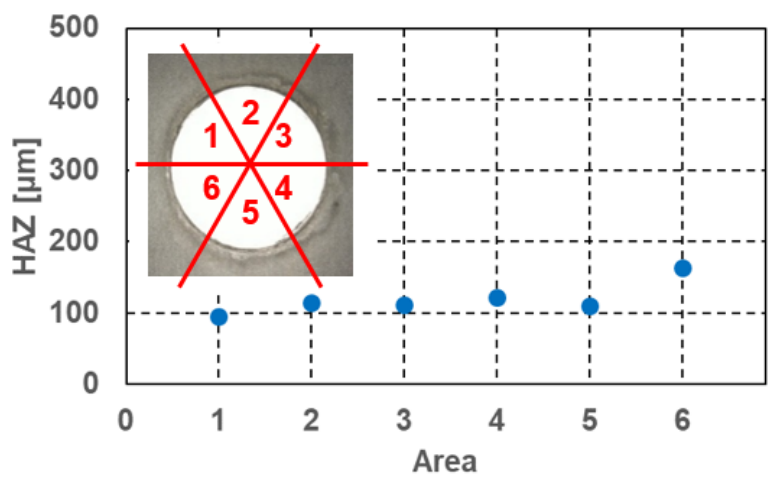

Fig. 8 Maximum width of HAZ in each area.

Figure 8 shows the maximum width of HAZ in each area. The HAZs were controlled to around $100 \mu \mathrm{m}$ in most areas and to $160 \mu \mathrm{m}$ even at the worst point. Although slight expansion of the HAZ in the direction of the carbon fiber was observed in area 6 , it is not a significant difference. 
Figure 9 shows the cross-sectional image of the drilled CFRP. No significant expansion of HAZ was observed even in the lower layers. The kerf angle was about 3 degree. It will result in a deviation of $0.2 \mathrm{~mm}$ on a diameter. The acceptable deviation of a diameter for riveting is said to about $0.4 \mathrm{~mm}$ in some applications. Therefore, holes made by our setup are suitable for precise machining. Figure 10 shows the images of the wall surface obtained by the optical microscope and the SEM. Although surface irregularities were observed in some regions, the surface condition was basically good. The drilling of the CFRP plate using our single-mode fiber laser enabled reductions in HAZ width and the achievement of a good wall surface, even at as short processing time as in the mechanical processing.

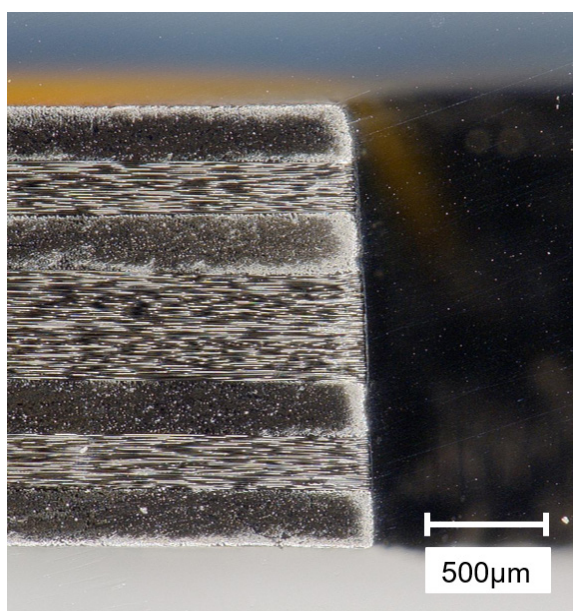

Fig. 9 Cross-sectional image of the processed CFRP.

(a)

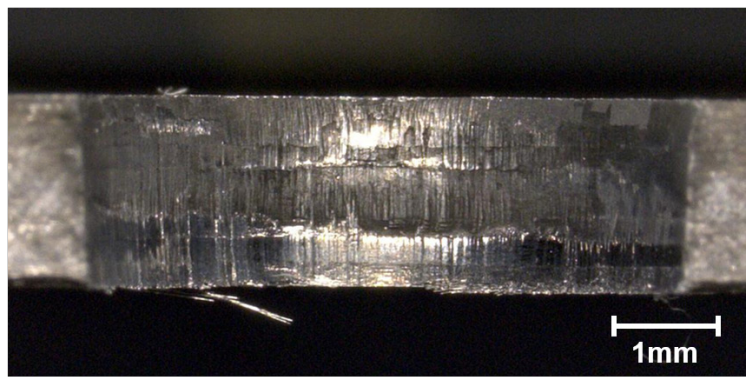

(b)

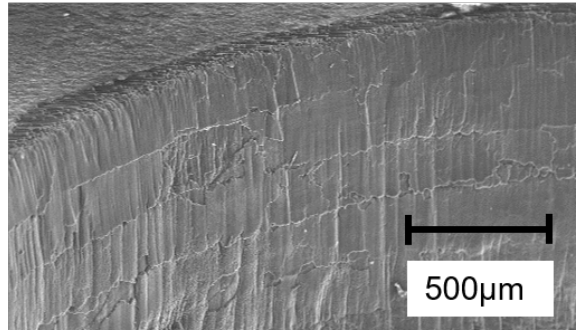

Fig. 10 Cross-sectional surface images obtained by (a) optical microscope and (b) SEM.

\section{Summary}

The 2-mm-thick CFRP plate has been successfully drilled with the in-house $3-\mathrm{kW}$ single-mode fiber laser and the galvanometer scanner. A processing time of 2.7 seconds has been achieved at a scanning speed of $10 \mathrm{~m} / \mathrm{s}$ and the interval of $20 \mathrm{~ms}$. The width of HAZ was reduced to about $100 \mu \mathrm{m}$ in all directions, and a good wall surface was achieved. The results show the potential of the high power CW single-mode fiber laser for the application to high speed and high quality drilling of the CFRP, as well as cutting.

\section{References}

[1] C. Emmelmann, M. Petersen, A. Goeke, and M. Canisius: Physics Procedia, 12, (2011) 565.

[2] J. Walter, M. Hustedt, R. Staehr, S. Kaierle, P. Jaeschke, O. Suttmann, and L. Overmeyer: Physics Procedia, 56, (2014) 1153.

[3] S. Bluemel, P. Jaeschke, O. Suttmann, and L. Overmeyer: Physics Procedia, 56, (2014) 1143.

[4] S. Bluemel, S. Bastick, R. Staehr, P. Jaeschke, O. Suttmann, and L. Overmeyer: Physics Procedia, 83, (2016) 328.

[5] R. Staehr, S. Bastick, S. Bluemel, P. Jaeschke, O. Suttmann, J.-P. Negel, V. Angrick, C. Stolzenburg, S. Kaierle, and L. Overmeyer: J. Laser Appl., 31, (2019) 022207.

[6] F. Schneider and D. Petring: Laser Technik Journal, 2, (2018) 61.

[7] A. Klotzbach, M. Hauser, and E. Beyer: Physics Procedia, 12, (2011) 572 .

[8] J. Stock, M. F. Zaeh, and M. Conrad: Physics Procedia, 39, (2012) 161.

[9] A. N. Fuchs, M. Schoeberl, J. Tremmer, and M. F. Zaeh: Physics Procedia, 41, (2013) 372.

[10] M. Oberlander, M. Canisius, P. Hergoss, K. Bartsch,D. Herzog, and C. Emmelmann: J. Laser Appl., 30, (2018) 032204.

[11] Y. Takubo, S. Ikoma, K. Nakayama, Y. Umeda, M. Kashiwagi, and D. Tanaka: The 38th International Congress on Applications of Lasers \& Electro-Optics (ICALEO), Orlando, (2019) Macro 502.

[12] M. Li, S. Li, G. Gan, Y. Zhang, Z. Liang, and X. Yang: J. Laser Appl., 31, (2019) 012004.

[13] A. F. Riveiro, F. Quintero, F. Lusquiños, J. del Val, R. Comesaña, M. Boutinguiza, and J. Pou: Composites Part A, 43 (2012) 1400.

[14] Y. Sato, M. Tsukamoto, F. Matsuoka, T. Ohkubo, and N. Abe: App. Surf. Sci., 417, (2017) 250.

[15]D. Herzog, M. Schmidt-Lehr, M. Oberlander, M. Canisius, M. Radek, and C. Emmelmann: Materials \& Design, 92, (2016) 742.

(Received: June 25, 2020, Accepted: October 8, 2020) 